\title{
Two-dimensional Material Membranes for Gas Separation
}

\author{
Mostapha Dakhchoune* \\ §SCS-DSM Award for best poster presentation in Inorganic Chemistry
}

\begin{abstract}
Two-dimensional (2D) materials with atom- or few-atoms-thick layers have emerged as building-blocks in the synthesis of the next-generation membrane-based separations. Generally, 2D material-based membranes display high permeation and high selectivity due to their unique structure composed of nanopores and nanochannels with extremely short transport pathways. In this review, the latest advances and ground-breaking research studies on 2D nanosheets for gas separation are highlighted with a focus on the different strategies in synthesizing 2D nanosheets, their assembly into thin membranes and the type of transport mechanism taking place in such membranes.
\end{abstract}

Keywords: Gas separation membranes · Two-dimensional materials · Two-dimensional nanosheets · Ultrathin membrane

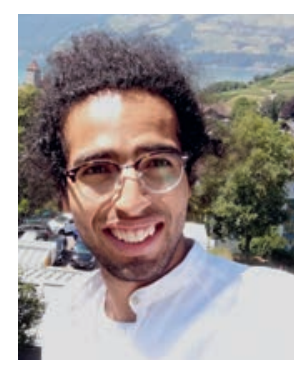

Mostapha Dakhchoune was born in Morocco in 1989. He obtained his Master's degree in Chemical and Process Engineering with honors at the University of Bologna (Italy). He conducted his Master thesis at KAUST University (Saudi Arabia) under the supervision of Prof. G.C. Sarti and Prof. I. Pinnau with his thesis focusing on polyimides polymeric membranes for gas separation. He is now a $\mathrm{PhD}$ candidate at École Polytechnique Fédérale of Lausanne (EPFL) in the Laboratory of Advanced Separations (LAS) supervised by Prof. K.V. Agrawal. His current research focuses on nanoporous two-dimensional silicates for gas separation.

\section{Introduction}

The separation of mixtures in the chemical industry plays an important role in the overall operating and capital plant cost. ${ }^{11]}$ Often, the plant core that produces the desired product accounts only for a small fraction of the cost compared to the following train of separations. This motivates the search for energy-efficient separations. ${ }^{[2]}$ In most systems, mixing is a favorable and spontaneous process and the separation of compounds is a non-spontaneous process that requires energy. Traditional technologies to overcome the thermodynamic disadvantage use heat as its driving force (e.g. distillation, absorption, and evaporation), making the overall separation processes energetically very expensive. Such technologies account for a significant portion of the world's energy consumption (10-15\%). [3]

Membrane-based separation technology has the potential to drastically cut down separation energy and has also inherent advantages such as small environmental footprint, continuous operation, and a simple modular design ideal for the remote off-shore separations. Nevertheless, membranes suffer from the well-known trade-off between permeance (i.e. the flux normalized by the transmembrane pressure difference) and selectivity. ${ }^{[4]}$ The advances in developing the ideal membrane (with both high permeance and selectivity) have been hampered by the low intrinsic performance displayed by conventional polymeric materials, and the fact that the geometrical configuration of threedimensional networks of entangled polymeric chains that form a selective layer limits the production of extremely thin defect-free selective layers.

The Nobel-prize-awarded discovery of graphene by Geim and Novoselov in 2004 triggered an intensive research interest onto two-dimensional (2D) materials for gas and liquid separations. Among these, 2D nanosheets are attracting increasing interest. ${ }^{[5]}$ The assembly of 2D nanosheets into thin films as building blocks makes them promising candidates for the next generation of membranes. The atom or few-atoms-thick nanosheets may lead to higher fluxes since they minimize the diffusional path that a molecule travels to cross the membrane thickness. Moreover, their in-plane crystalline structure with highly-ordered pore arrays ensures high size-sieving selectivity. One of the most studied class of two-dimensional membranes is based on graphene and graphene derivatives, such as graphene oxide $(\mathrm{GO})$ and reduced graphene oxide (rGO). These kinds of membranes have shown extensive applications in water and gas separation. ${ }^{[6]}$ However, the in-plane crystalline honeycomb structure of graphene is impermeable even to the smallest gas molecules due to the electron density of the aromatic rings. This suggests that the sole transport taking place during separations is through the nano-channels between nanosheets and through defects originating from the exfoliation of $\mathrm{GO} / \mathrm{rGO}^{\left[{ }^{[7]}\right.}$ Nanosheets with well-ordered pore networks running parallel to the gas concentration gradient would give rise to high permeance membranes, while the uniform pore size ensures high selectivity.

2D nanosheet-based membranes can be classified in two main categories based on their transport mechanism: i) intrinsically non-porous nanosheets which rely on many strategies to finetune the selective interlayer spacings (GO and $\mathrm{rGO},{ }^{[8]}$ MXenes, ${ }^{[9]}$ transition metal dichalcogenides, TMDs, ${ }^{[10]}$ and layered double hydroxides, or $\operatorname{LHDs}^{[11]}$ ); and ii) porous nanosheets where the transport of the molecules occurs parallel to the nanosheet thick- 
ness and through their pores (nanoporous graphene, ${ }^{[12]}$ zeolites, ${ }^{[13]}$ metal-organic frameworks MOFs, ${ }^{[14]}$ covalent organic frameworks COFs, ${ }^{[15]}$ and graphitic carbon nitrides ${ }^{[16]}$ ).

This review looks at the recent advances and ground-breaking research studies on 2D nanosheets for gas separation. It focuses on the different strategies in synthesizing 2D nanosheets, their assembly into thin membranes and the type of transport mechanism present in such membranes.

\section{Synthesis and Separation Mechanism of 2D Nanosheets}

The synthesis of 2D nanosheets with nanometer or subnanometer thickness will remarkably reduce the transport resistance with increased fluxes. Two major routes, top-down and bottomup, are utilized to synthesize 2D nanosheets. The former relies on the difference between the in-plane and out-of-plane forces that keep atoms and layers together. Typically, weak Van der Waals interactions holding together stacked nanosheets can be overcome by different strategies such as ultrasound,[17] solventassisted exfoliation, ${ }^{[18]}$ polymer intercalation coupled with shearing stress, ${ }^{[13]}$ and freeze and thaw ${ }^{[19]}$ exfoliation separating the single- or few-layered nanosheets. Restacking of the nanosheets, low exfoliation yields, and fragmentation represent some of the major drawbacks in the top-down strategy. ${ }^{[20]}$ The bottom-up route relies on the suppression of the orthogonal crystal growth either by reaction nanoconfinement (e.g. air-liquid and liquid-liquid interfaces, ${ }^{[21,22]}$ nanoreactors $\left.{ }^{[23]}\right)$ or by the use of third-party molecules (e.g. surfactants) which direct the growth in the in-plane domains. ${ }^{[24]}$ The need for substrates, interfaces, and surfactants hinders mass production in the bottom-up approach. Moreover, the resulting nanosheets are still relatively thick $(5-50 \mathrm{~nm}) .{ }^{[25]}$

The selection of either route depends on which features the nanosheets need to possess for the desired application. Both synthesis strategies can originate lateral sizes larger than hundreds of $\mu \mathrm{m},{ }^{[22,26]}$ and their assembly into membranes can be performed by several methods, such as pressure-assisted filtration, ${ }^{[27]}$ spray coating, ${ }^{[28]}$ casting, ${ }^{[29]}$ layer-by-layer deposition, ${ }^{[30]}$ and hot-drop coating. ${ }^{[17]}$ The selective pathways that transport the gas molecules vary depending on the structural nature of the nanosheets which can be porous (e.g. zeolites, MOFs, COFs, etc.) or nonporous (e.g. GO, TMDs, LHDs, etc.). The controlled stacking of the latter type of nanosheets produces nanochannels due to the interlayer repulsive interactions. The intercalation of guest molecules between the layers, besides creating empty interlayer pathways for the molecules, can facilitate their transport based on the different affinity between the guest molecules and the species being separated. ${ }^{[31,32]}$ Even though membranes of a few nanometers in thickness can be fabricated, the distance traveled by a molecule in crossing the whole membrane thickness can be orders of magnitude longer, resulting in lower fluxes. To shorten the molecule transport pathways, the use of intrinsically porous nanosheets allows perpendicular transport along the gas gradient concentration minimizing the transport resistance and resulting in higher productivity.

Herein, the latest advances and major breakthroughs in 2D nanosheets-based gas separation will be highlighted. The first part of this review begins with porous nanosheets where the inplane porous structure plays an important role in the separation, while the second part will describe nonporous nanosheets-based membranes where the main focus is on fine-tuning the interlayer spacings to tune the separation performance.

\section{Nanoporous Transport}

\subsection{Zeolites}

Zeolites are alumino-silicates with a tetrahedrally connected $\mathrm{SiO}_{2}$ network forming well crystalline domains with pores ranging from 0.25 to more than $1 \mathrm{~nm} \cdot{ }^{[33]}$ Because of their unique properties of well-defined pores, high porosity, chemical, and mechanical stability, zeolites have been used in many chemical processes. ${ }^{[34]}$ However, applications as membranes have seen only the LTA-type of zeolite implemented in the dehydration of bioethanol. ${ }^{[35]}$ To avoid crystallization defects in the polycrystalline zeolite membranes, thicknesses up to tens of micrometers are required resulting in a much higher transport resistance. In this regard, the use of $2 \mathrm{D}$ zeolite nanosheets as building-blocks for the gas separation has attracted much attention as high-performance molecular sieving membranes.

Nanosheet crystallinity, uniform thickness, and high aspect ratio play a major role in the fabrication of high-performance membranes. The solution-based exfoliation technique, dominated by sonication to break the interlayer interactions, could not meet these criteria. The resulting zeolite nanosheets have too many defects to make them suitable for membrane fabrication. Tsapatsis and coworkers developed the use of the polymer-melt-compounding exfoliation technique for ITQ-1 and multilamellar silicalite-1, resulting in high-quality MWW and MFI nanosheets, respectively.[13] After the purification steps to isolate the nanosheets from the polymeric matrix and removal of larger unexfoliated particles by centrifugation, the 1 or 1.5 unit-cell-thick zeolite nanosheets were assembled into thin membranes by vacuum filtration. Even though they were successful in fabricating well b-oriented MFI nanosheets membranes (c-oriented for MWW) which exposes the shape-sieving $0.55 \mathrm{~nm}$ pore of MFI, no separation for the xylene isomers was reported due mainly to the presence of non-selective pathways between the nanosheets (Fig. 1a). After performing a mild secondary growth step to seal these defects, the prepared membranes showed a $p$-xylene/o-xylene separation factor (SF) of 40 to 70 and $p$-xylene permeance of $3 \times 10^{-7} \mathrm{~mol} \mathrm{~m}^{-2} \mathrm{~s}^{-1} \mathrm{~Pa}^{-1}$ at $150{ }^{\circ} \mathrm{C}$. The final membrane thickness was only $200 \mathrm{~nm}$, which is an order of magnitude thinner than conventional polycrystalline zeolite membranes.

The employment of secondary growth is considered to be a problematic step because it is an expensive and complicated process that hinders the scaling up for commercial applications and it highly affects the membrane's reproducibility. ${ }^{[36]}$ To bypass the solvothermal recrystallization step, the same group developed a gel-less secondary growth to seal non-selective pathways. MFI nanosheets were deposited on a porous Stöber silica layer by vacuum filtration, and a gel-less secondary growth was done. The resulting total membrane thickness was $100 \mathrm{~nm}$. The Stöber spheres acted as a silica source during the regrowth and the same b-orientation was maintained, resulting in a higher $p$-xylene/oxylene SF compared to the previous study maintaining similar fluxes. ${ }^{[37]}$ Another key step to improve the performance further was the partial removal of the organic structure-directing agent, which allowed the dispersion of the nanosheets in more friendly solvents such as water and ethanol, and offered the opportunity to stabilize the nanosheets at the air/water interface. The stabilization of monolayers of MFI nanosheets in the air/water interface of a conical reservoir, along with the slow withdrawal of water from the bottom part allowed the careful deposition of the nanosheets onto a porous Stöber silica layer, giving rise to very thin zeolite nanosheet-based films (Fig. 1b). Subsequent exposure of the nanosheets layer to a gel-less secondary growth process resulted in an unprecedented separation performance with a SF of the $p$-xylene/o-xylene over 10000 with a permeance of $2.9 \times 10^{-7} \mathrm{~mol}$ $\mathrm{m}^{-2} \mathrm{~s}^{-1} \mathrm{~Pa}^{-1}$.[30,38]

Bottom-up synthesis of MFI nanosheets can eliminate the structural defects that may arise from the top-down approach. Tsapatsis and coworkers achieved a breakthrough in synthesizing 5 nm-thick MFI nanosheets (2.5 unit cells) by appropriately suppressing the orthogonal growth with the use of bis-1,5(tripropyl ammonium)pentamethylene diiodide structure-directing agent (Fig. 1c). The pioneering work produced micrometer MFI nanosheets in lateral size, and their stacking onto a porous support 

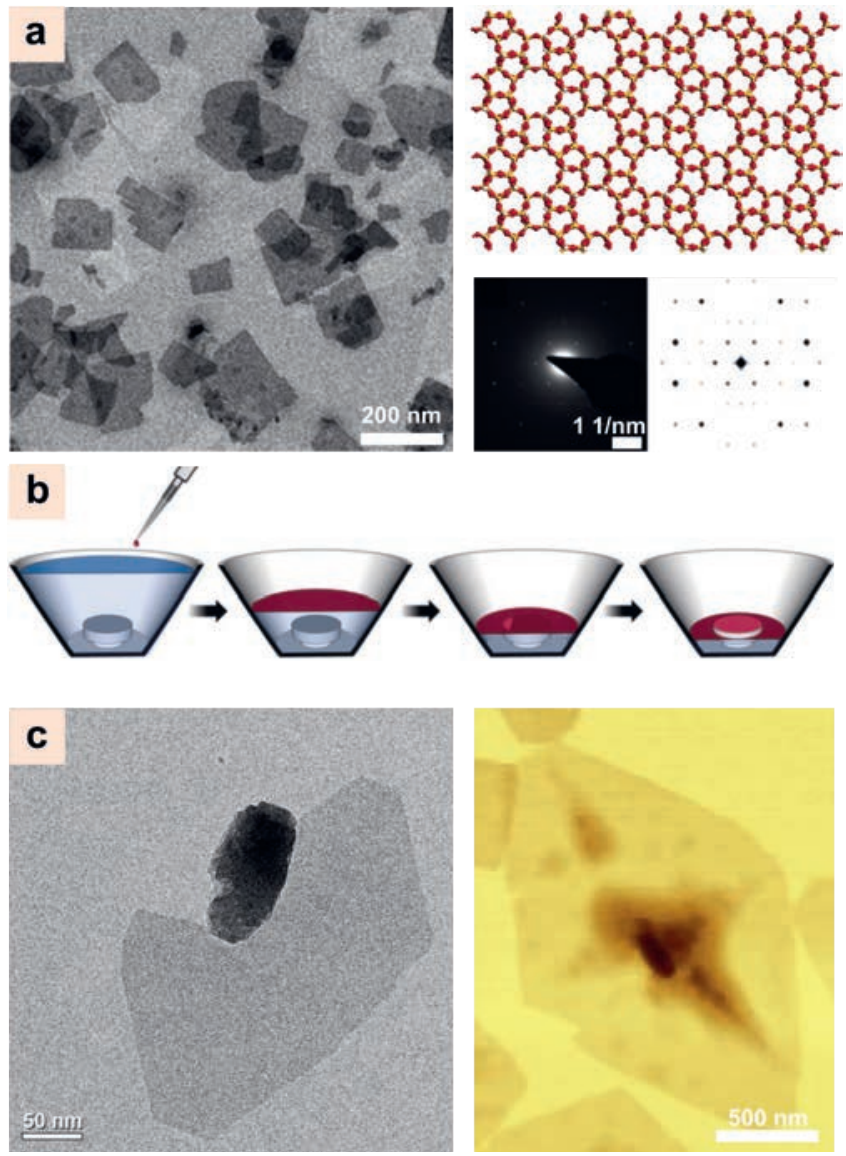

Fig. 1. (a) TEM image of a single MFI nanosheet and its crystal structure along the $b$ axis. Electron diffraction pattern measured and simulated of MFI. ${ }^{[13]}$ (b) Illustration of MFI monolayer deposition in a conical reservoir. The liquid is slowly drained from the bottom to lower the level and compress the nanosheets. ${ }^{[38]}$ (c) TEM and AFM images. Gradual and complete bottom-up growth of the MFI nanosheet around the seed. ${ }^{[66]}$ Reprinted with permission. Ref. [13]: Copyright 2011, American Association for the Advancement of Science. Ref, [66]: Copyright 2017, John Wiley and Sons.

with the previously developed gel-less secondary growth resulted in excellent performance for the xylene isomers separation. [39]

Since the first synthesis of 2D membranes with MFI and MWW nanosheets, extensive work has been done by Tsapatsis and coworkers in developing very thin nanosheet layers without the need for solvothermal secondary growth. However, more research needs to be performed in this direction to completely eliminate the gel-less secondary growth. Moreover, increase in the exfoliation yields, synthesis of larger lateral nanosheet size, and the use of cheaper supports would facilitate the scale-up of these high-performance membranes.

\subsection{MOFs}

Metal-organic frameworks (MOFs) are a new emerging class of materials consisting of metal ions or clusters coordinated with organic linkers, leading to a highly-crystalline nano- or microporous network. Due to their high porosity, tunable pore size and structure, and abundant surface functional groups, they have attracted an exponential interest in many applications, such as separation and gas storage, catalysis, chemical sensing, and drug delivery. ${ }^{[40]}$ Besides their three-dimensional structure, many layered MOFs have been reported in literature offering the potential of their implementation as building blocks for 2D-based membranes. ${ }^{[11]}$ Nevertheless, challenges remain in the top-down exfoliation strategy to fully preserve the nanosheets' crystallinity and their high aspect ratio. On the contrary, the bottom-up approach could produce higher quality MOF nanosheets.
Despite the challenges in the top-down approach in preserving the morphological and structural integrity of the MOF nanosheets, Yang's group was successful in exfoliating 1-nm thick MOF nanosheets with a lateral size larger than a micrometer while preserving their crystallinity. ${ }^{[17]}$ They used the combination of a low-energy wet-ball milling and sonication to exfoliate the MOF precursor $\mathrm{Zn}_{2}-(\text { bim) })_{4}$ (bim=benzimidazole). Their key finding was to use a mixture of solvents, namely methanol and propanol. The former would access the interlayer spacing, thanks to its smaller kinetic diameter, while the latter was able to stabilize the exfoliated nanosheets. The exfoliated MOF nanosheets have a theoretical pore size of $0.21 \mathrm{~nm}$ but in practice it is larger due to the flexibility of the MOF structure. To avoid the restacking of the nanosheets, they adopted the hot-drop coating method onto a ceramic porous support which increases the turbostratic disorder between the nanosheets (Fig. 2a). Ultrathin membranes (thickness $<10 \mathrm{~nm}$ ) were fabricated, which gave sharp molecular cut-off between $\mathrm{H}_{2}$ and $\mathrm{CO}_{2}$ with $\mathrm{SF}$ as high as 291 with a $\mathrm{H}_{2}$ permeance higher than $2000 \mathrm{GPU}\left(1 \mathrm{GPU}=3.3 \times 10^{-10} \mathrm{~mol} \mathrm{~m}^{-2} \mathrm{~s}^{-1} \mathrm{~Pa}^{-1}\right)$. Interestingly, they observed an unusual increase in selectivity along with the increase of $\mathrm{H}_{2}$ permeance. They explained that the restacking of the nanosheets could have a detrimental effect on the $\mathrm{H}_{2}$ transport while having only a negligible effect on the $\mathrm{CO}_{2}$ permeance, hence increasing the in-plane disorder of the nanosheets would expose the pores of the nanosheets to the gas phase. Due to the delicate nature of their system, it is worth mentioning that
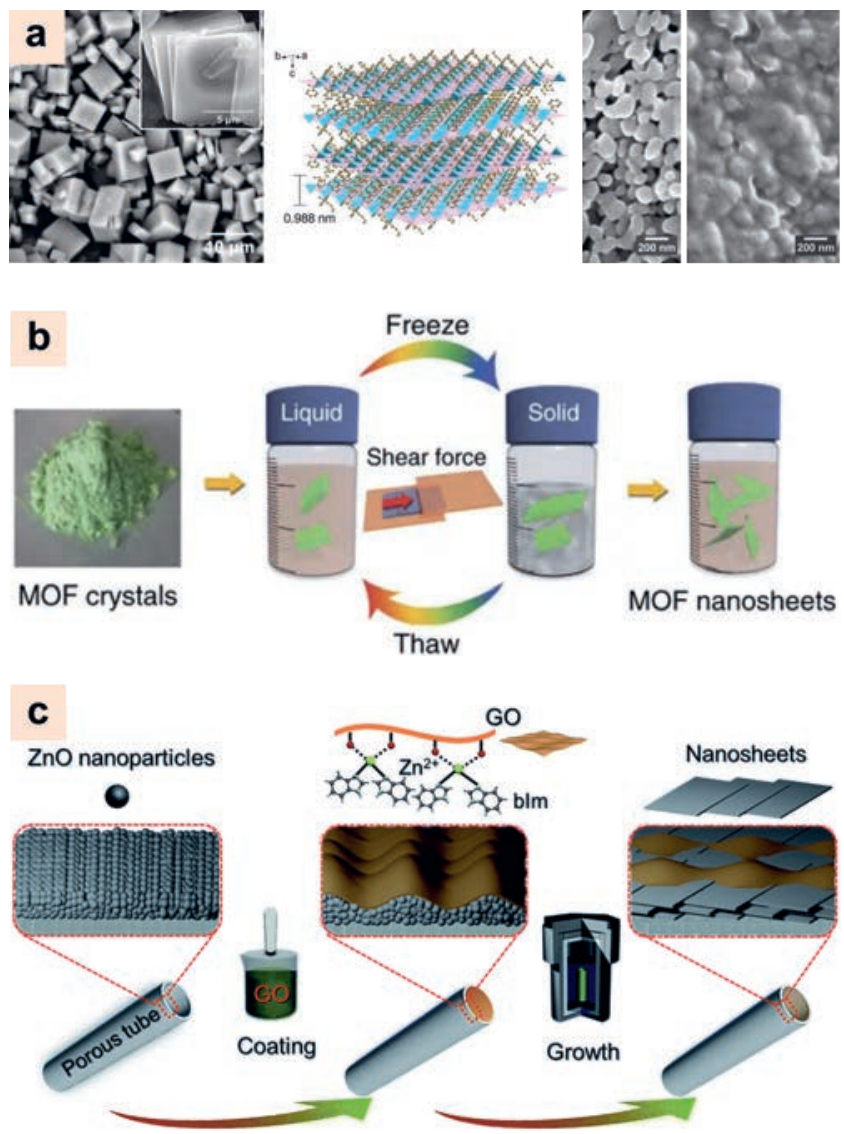

Fig. 2. (a) SEM image of as-synthesized $\mathrm{Zn}_{2}(\mathrm{bim})_{4}$ with its theoretical layered structure and the SEM images of bare porous support as well as with coated nanosheets. ${ }^{[14]}$ (b) Image of MAMS-1 crystal with an illustration of the freeze-thaw exfoliation method. ${ }^{[19]}$ (c) Illustration of the steps for preparing oriented $\mathrm{Zn}_{2}(\mathrm{bim})_{4}$ nanosheets membrane by zinc oxide self-conversion growth in GO confined space. ${ }^{[42]}$ Reprinted with permission. Ref. [14]: Copyright 2014, American Association for the Advancement of Science. Ref. [19]: Open access, Copyright 2017 Springer Nature. Ref. [42]: Open access, The Royal Society of Chemistry (RSC). 
no absolute pressure difference across the membrane was applied since it would cause the bending/deformation of the nanosheets.

The ball milling and ultrasound exfoliation might damage fragile MOFs. Wang et al. employed a mild freeze and thaw technique to exfoliate MAMS-1 (Mesh Adjustable Molecular Sieve, $\mathrm{Ni}_{8}(5-$ bbdc $)_{6}(\mu-\mathrm{OH})_{4}, 5$-bbdc stands for 5-tert-butyl-1,3-benzenedicarboxylate) into nanosheets. ${ }^{[19]}$ The fast volumetric expansion of the solvents during the thawing process created shear stress between the layers overcoming the weak Van der Waals interactions (Fig. 2b). MAMS-1 possesses two different pore domains: $0.29 \mathrm{~nm}$ pore aperture parallel to nanosheet plane and $0.55 \mathrm{~nm}$ aperture perpendicular to the smaller pore. It is then of vital importance to ensure the right in-plane orientation of the nanosheet to expose the more selective pore. This could be achieved using the hot-drop casting method at a temperature slightly higher than the solvent boiling point to allow fast evaporation and avoiding the restacking of nanosheets as well as the desired nanosheets orientation. Membranes with varying thicknesses were fabricated up to 40 nm. The 12-nm membranes displayed a $\mathrm{H}_{2} / \mathrm{CO}_{2} \mathrm{SF}$ of 34 and $\mathrm{H}_{2}$ permeance of $6516 \mathrm{GPU}$, while for the slightly thicker membrane they could achieve a higher separation factor of 235 at the expense of an order of magnitude lower $\mathrm{H}_{2}$ permeance. Interestingly, their membranes showed a reversed thermo-switchable behavior. The gas pair selectivity was ranging from 5 at $100{ }^{\circ} \mathrm{C}$ to 245 at room temperature. In situ X-ray measurements helped explain this abnormal behavior with the flexibility of the tert-butyl groups as a function of temperature. This stimuli-responsive feature finding can be potentially exploited in temperature-swing related gas separations.

Membranes supported on small-diameter tubular supports are of great importance in gas separation applications because they offer high surface area per unit of volume and they can withstand higher pressures. However, the top-down approach is confronted with many engineering challenges in fabricating 2D nanosheets onto tubular supports. On the other hand, it is not trivial to directly grow 2D MOF membranes on tubular substrates. Li et al. were the first to report the synthesis of a crystalline nanosheet membrane on a porous tubular substrate by direct growth. ${ }^{[42]}$ They exploited the GO-guided growth by first depositing a thin layer of $\mathrm{ZnO}$ nanoparticles on the tubular support by dip-coating, followed by another layer of GO upon which the nanoparticles self-converted into $\mathrm{Zn}_{2}(\mathrm{bIm})_{4}$ nanosheets membranes. The $200 \mathrm{~nm}$ thin membranes displayed a $\mathrm{H}_{2} / \mathrm{CO}_{2}$ selectivity of 106 and a $\mathrm{H}_{2}$ permeance of ca. $500 \mathrm{GPU}$ (Fig. 2c). Their strategy offers the opportunity for the large-scale fabrication of highly-oriented MOF nanosheet membranes on a tubular geometry, which is relevant for industrial gas separations.

Unprecedented performance has been achieved with 2D MOF nanosheets-based membranes. Nevertheless, much effort needs to be done before achieving the precise manipulation of the nanosheets, especially at large scale. Fabrication of nanosheet lateral sizes larger than $1 \mu \mathrm{m}$, uniform nanosheet thickness, higher degree of crystallinity (particularly for the top-down exfoliation approach), scalable membrane fabrication strategies are all vital features that still represent a hurdle in the commercialization of MOFs membranes. Moreover, investigations on their mechanical and chemical stability in real commercial operating conditions need to be performed.

\subsection{COFs}

Covalent organic frameworks (COFs) were first discovered by Yaghi and coworkers in 2005.[15] They are composed of solely covalently-bonded organic linkers displaying highly crystalline networks with pore size ranging from $0.5 \mathrm{~nm}$ up to $4.7 \mathrm{~nm} \cdot{ }^{[43]}$ They have attracted increasing attention as membrane materials. Simulation studies concluded that exfoliation from their 3D structure is possible and that their separation performance is highly promising. ${ }^{[44,45]}$ Although most of the pore sizes found in COFs are larger than the kinetic diameters of most gases (He $2.6 \AA, \mathrm{H}_{2}$ $2.9 \AA, \mathrm{N}_{2} 3.6 \AA, \mathrm{CO}_{2} 3.3 \AA$, and $\mathrm{CH}_{4} 3.8 \AA$ ), the effective pore aperture exposed to the gas phase can be reduced by pores overlapping. Reports of single-phase COF membranes that show interesting separation performance for gases remain elusive to-date. Li et al. successfully fabricated 2D COF-1 nanosheets starting from the parental 3D structure via solvent-assisted exfoliation. ${ }^{[18]}$ The nanosheets were assembled into $100-n m$ thin membranes by drop-coating at room temperature. Nevertheless, due to the pore size larger than $1 \mathrm{~nm}$, the membrane displayed a $\mathrm{H}_{2} / \mathrm{N}_{2}$ selectivity of $c a .4$.

An interesting strategy to overcome the pore size limitation of $\mathrm{COFs}$ is to assemble them in a way that the pores in one COF layer are partially blocked by another COF layer effectively reducing the size of the pores available for the transport of gases. Caro and coworkers recently synthesized bilayer COF-COF composite membranes by successively regulating the growth of imine-based COF-LZU1 and azine-based ACOF-1 via a temperature-swing solvothermal approach. ${ }^{[46]}$ The resultant bilayer COF-LZU1ACOF-1 synthesized on a flat porous support displayed a much higher gas separation performance compared to the single phased for each $\mathrm{COF}$ due to the formation intercalated pore networks of $0.3-0.5 \mathrm{~nm}$.

The incorporation of COFs nanosheets into polymeric membranes (Mixed Matrix Membranes, MMMs) was demonstrated to have a synergistic effect improving the polymer performance. Zhao's group synthesized water-stable 2D NUS-2 and NUS-3 COFs with both thermal and chemical resistance to basic and acidic environments. ${ }^{[47]}$ The COF nanosheets were dispersed in a continuous phase of polyetherimide (PEI) and polybenzimidazole (PBI) polymers to form mixed matrix membranes. For instance, the NUS-2@PBI membranes with a NUS-2 loading of $20 \%$ displayed a $\mathrm{H}_{2} / \mathrm{CO}_{2}$ selectivity of 31.4 surpassing the 2008 Robeson upper bound. Although the flux increased along with the selectivity, the achieved values were too low for commercial applications.

COFs crystallinity, ordered porous structure, large surface area, and their wide-range of pore sizes make them excellent candidates for a variety of applications. However, the synthesis of COFs with pore sizes less than $1 \mathrm{~nm}$ remains a challenge limiting their application for gas separation. Moreover, studies on longterm stability of COFs membranes need to be addressed.

\subsection{Nanoporous Graphene}

Graphene, the single layer of graphite, has attracted tremendous attention in the last decade because of its carbon atom-thick layer, mechanical strength, chemical inertness, and its impermeable nature to the smallest gas molecules due to the delocalized electronic cloud of the $\pi$-orbitals. ${ }^{[48]}$ Simulation studies have shown that if nanopores are present in the graphene lattice its potential for both water desalination and gas separation is very high. They predicted orders of magnitude higher performance compared to actual commercial membranes. ${ }^{[49,50]}$ Motivated by these findings, an extensive amount of work has been done to develop strategies to selectively create nanopores in the honeycomb graphene lattice, such as focused-electron and ion beam bombardment, ${ }^{[51,52]}$ oxygen plasma, ${ }^{[53]}$ and ultraviolet-induced oxidative etching. ${ }^{[54]}$

Great advances have been achieved in the development of nanoporous graphene membranes but there is still a lot to be done. Current technologies to etch the pores cannot satisfy three conditions required to exploit the full potential of single layer nanoporous graphene membranes: i) accurate pore size control in the range of $0.2-1 \mathrm{~nm}$; ii) narrow pore distribution, and iii) a high pore density. In general, it is the trade-off between the three conditions that limits experimental results in graphene membranes to match the predicted performances by the simulation studies. Other chal- 
lenges in the preparation and scale-up of graphene membranes are the formation of intrinsic defects during the chemical vapor deposition (CVD) used to crystallize the graphene layer, as well as extrinsic defects formed during the transfer of the graphene layer from the metallic substrate to the porous one. ${ }^{[55]}$

Regardless of the challenges, recent breakthroughs have allowed exploration of the potential of single-layer graphene for membranes. For example, Surwade et al. suspended singlelayer graphene on a $5-\mu \mathrm{m}$ silicon-etched hole, and were able to etch pores in the range of $0.5-1 \mathrm{~nm}$ with oxygen-plasma. They achieved a water vapor flux as high as $10^{6} \mathrm{~g} \mathrm{~m}^{-2} \mathrm{~s}^{-1}$ and when the membrane was tested for water desalination, a nearly $100 \%$ salt rejection was achieved. ${ }^{[53]}$ Moreover, our group has recently reported a new nanoporous carbon-film-based transfer technique, which circumvents the removal of mechanically reinforcing polymers. ${ }^{[12]}$ The use of high-flux nanoporous carbon film guaranteed mechanical reinforcement to the graphene monolayer without the need for removal. A $1 \mathrm{~mm}^{2}$ of single-layer graphene was transferred onto a porous tungsten support, allowing the evaluation of transport properties from intrinsic defects. $\mathrm{H}_{2}$ permeance of 1300 GPU and $\mathrm{H}_{2} / \mathrm{CH}_{4}$ selectivity of 25 were achieved. After exposing the same membrane to ozone functionalization-based etching, the $\mathrm{H}_{2}$ permeance and $\mathrm{H}_{2} / \mathrm{CH}_{4}$ selectivity increased by 300 and $150 \%$, respectively. To have more control over the pore density and size, Zhao et al. developed a synergistic, partially decoupled defect nucleation and pore expansion strategy using $\mathrm{O}_{2}$ and $\mathrm{O}_{3}$ treatment. They achieved a high pore density of $c a .2 .1 \times 10^{12} \mathrm{~cm}^{-2}$ and $\mathrm{H}_{2}$ permeance from 1340 to $6045 \mathrm{GPU}$ and $\mathrm{H}_{2} / \mathrm{CH}_{4}$ selectivity from 15.6 to $25.1 .{ }^{[56]}$

Nanoporous graphene with its carbon-atom thickness and tunable pore size is a strong candidate for the next generation of membranes. However, more research needs to be performed to address the major issues that limit scalability. Angstrom-precise pore-size control, narrow pore size distribution, high-pore density, and a more scalable transfer strategy are the major bottlenecks for its commercialization.

\section{Nanochannels Transport}

\subsection{Graphene Oxide}

GO is a derivative of graphene and it is widely obtained from the oxidation of graphite using Hummer's method. ${ }^{[57]}$ The oxidation method developed by Hummer produces GO with a high content of oxygen-rich functional groups (hydroxyl, carboxyl, and epoxy groups) on the basal plane as well as on the nanosheet's perimeter. Unlike nanoporous graphene, the transport of molecules occurs mainly through the gallery spacings, and through in-plane defects/gaps generated during the exfoliation and/or membrane assembly.

The fine-tuning of the interlayer spacings between the nanosheets plays a key role in gas and water separations. Li et al. assembled GO nanosheets into a $1.8 \mathrm{~nm}$ membrane by simple vacuum filtration achieving unprecedented $\mathrm{H}_{2} / \mathrm{CO}_{2}$ and $\mathrm{H}_{2} / \mathrm{N}_{2}$ selectivities of 3400 and 900, respectively (Fig. 3a). However, the $\mathrm{H}_{2}$ flux was low for such a thin membrane demonstrating the 'zigzag' type of transport. ${ }^{[8]}$ The importance of rationally manipulating the repulsive/attractive forces between the functional groups in the nanosheets can drastically change the GO membrane performance. $\mathrm{Xu}$ and coworkers rationally designed external forces (i.e. vacuum-filtration-coupled shearing forces derived from the spin-coating system) as well as inner forces (i.e. using intercalating polymers) to finely manipulate the $2 \mathrm{D}$ channels aperture. ${ }^{[58]}$ The polymer intercalation suppressed the repulsive force, while the external contributed to the well-ordered packing of nanosheets. The as-prepared membranes, with a $2 \mathrm{D}$ channel of $0.4 \mathrm{~nm}$, displayed 2-3 orders of magnitude improvement in hydrogen permeance, and a 3 -fold enhancement in the $\mathrm{H}_{2} / \mathrm{CO}_{2}$ selectivity (Fig. 3b).
The interaction of the guest molecules with the functional groups present in the nanosheets can facilitate the transport of molecules. For example, $\mu \mathrm{m}$-thick GO membranes that were impermeable to even the smallest gases changed their behavior after being exposed to water. ${ }^{[7]} \mathrm{A}$ fast transport took place due to a combination of the favorable interaction between water molecules and the functional groups of GO, and the fact that water can flow frictionless in the hydrophobic domains of GO.[31] On a similar principle, Zhou et al. functionalized GO nanosheets with a brushlike $\mathrm{CO}_{2}$-philic agent (piperazine) using vacuum filtration on a tubular support. The 20 -nm thick membranes exhibited a remarkable $\mathrm{CO}_{2}$ permeance of $1020 \mathrm{GPU}$ and a $\mathrm{CO}_{2} / \mathrm{N}_{2}$ selectivity of 680 , while the non-grafted nanosheets showed orders of magnitude lower performance (Fig. 3c). ${ }^{32]}$

Considerable progress has been achieved in the fabrication methods of GO membrane as well as high performance. However, the long-term stability of the well-designed nanochannels, especially in the presence of water, along with the development of low defective nanosheets during exfoliation need to be fully investigated. Moreover, the inefficient 'zig-zag' type of transport motivates the formation of few-nanometer thick membranes to increase productivity, which might be technically challenging to engineer on an industrial large scale.

\subsection{Transition Metal Dichalcogenides (TMDs)}

TMDs are represented by the general chemical formula $\mathrm{MX}_{2}$ ( $\mathrm{M}$ is a transition metal such as Mo or $\mathrm{W}$ and $\mathrm{X}$ is a chalcogen such as S, Se, and Te) and they have attracted increasing attention in catalysis, sensor, electronic devices, etc. ${ }^{[10]}$ Similar to GO, TMDs are strongly bonded in-plane, while weaker interactions hold together the single layers. Chemical exfoliation, where the intercalation of lithium-based compounds followed by the strong reaction between lithium and water generates $\mathrm{H}_{2}$ gas, can overcome the interlayer interactions and yield single layer TMD nanosheets. Additionally, TMDs can also be directly grown by CVD synthesis. ${ }^{[10]}$

TMD nanosheet-based membranes have shown promising results for liquid separation applications but good performance for gas separation remains to be demonstrated. The performance of TMD nanosheet-based membranes in water is similar or even better than GO-based membranes. ${ }^{[59,60]}$ Wang et al. fabricated an ultrathin membrane $(17 \mathrm{~nm})$ by stacking chemically exfoliated $\mathrm{MoS}_{2}$ through vacuum filtration achieving a high permeance of more than 27000 GPU. ${ }^{[61]}$ However, the $\mathrm{H}_{2} / \mathrm{CO}_{2}$ selectivity was below Knudsen selectivity indicating the presence of non-selective channels. Interestingly, it was found that $2 \mathrm{D} \mathrm{MoS}$, undergoes phase transition upon heating going from an octahedral to a trigonal prismatic phase, which translates in a decrease of the interlayer spacings from 1.1 to $0.62 \mathrm{~nm}$, respectively. ${ }^{[62]}$ Surprisingly, membranes prepared from this type of phase-changed nanosheets displayed unvaried selectivity for the $\mathrm{H}_{2} / \mathrm{CO}_{2}$ pair gas selectivity while an increase of $30 \%$ in $\mathrm{H}_{2}$ permeance was witnessed. The authors attributed such behavior mainly to an interbundle-type of transport rather than from the single interlayer spacing.

TMDs represent a promising class of building-blocks for gas separation. However, deeper investigation on the tailoring of the nanochannels is required to obtain gas selective pathways.

\subsection{MXenes}

MXene was discovered by Gogotsi and coworkers in 2011, and since then it has been extensively implemented in many fields such as catalysis, water desalination, electronics, etc. ${ }^{[63]}$ MXenes are a class of transition metal carbides with generic formula MAX, where M is an early transition metal, $\mathrm{A}$ is an element from group 13 or 14 and $\mathrm{X}$ can be either $\mathrm{C}$ or $\mathrm{N}$. Their 2D form was obtained with the selective HF etching of the A layer resulting in a layered MX type of structure which can be further 

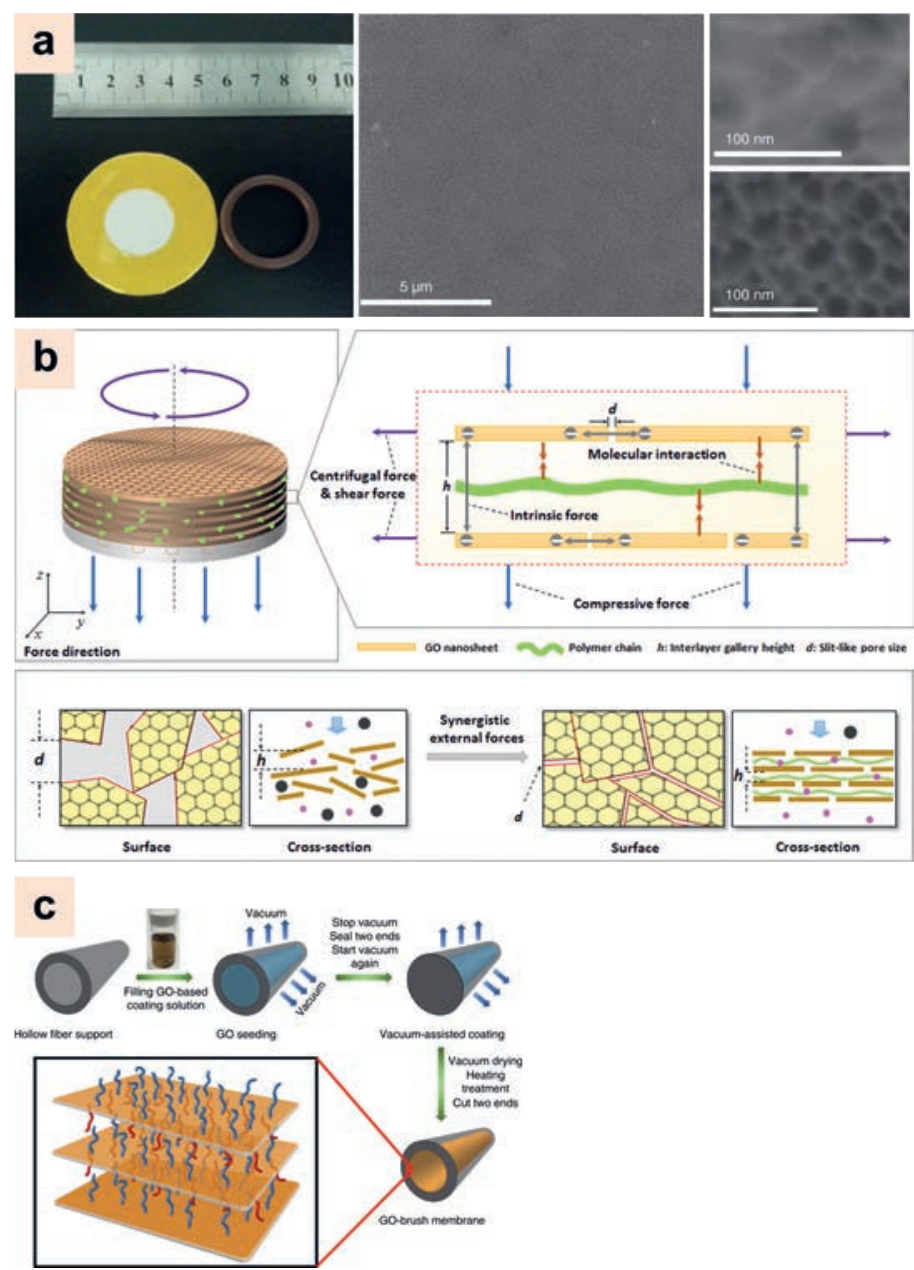

Fig. 3. (a) Picture of GO membrane on porous AAO support. Yellow and brown circles are Kapton tape and O-ring for sealing the membrane. Middle, SEM image of $18 \mathrm{~nm}$-thick GO membrane on AAO support. Right, SEM images of AAO support with (top) and without (bottom) GO nanosheets. ${ }^{[8]}$ (b) Illustration on the design of GO nanochannels with external forces application. Enlarged view shows resultant force (i.e., compressive, centrifugal and between the polymer and GO sheets). Below, hypothetical assembly of GO sheets in the surface and crosssection with (right) and without (left) the application of the rationally designed forces. ${ }^{[58]}$ (c) Illustration of the fabrication process of $\mathrm{CO}_{2}$-based hollow fiber membranes with a brush-like $\mathrm{CO}_{2}$-philic agent. Red and blue brushes are a visual aid to distinguish between molecules grafted on the bottom and top of the GO surface, respectively. ${ }^{\left[{ }^{[2]}\right.}$ Reprinted with permission. Ref. [8]: Copyright 2013, the American Association for the Advancement of Science. Ref. [58]: Copyright 2016, American Chemical Society. Ref. [32]: Open access, Copyright 2017 Springer Nature publisher.

mechanically exfoliated into single layers. ${ }^{[63,64]}$ MXenes are usually reported as $M_{n+1} X_{n} T_{x}$ where $T$ can be a hydroxyl, oxygen or fluorine group.

MXene nanosheets are impermeable and the transport is mainly a function of the interlayer spacings. The first application of 2D MXenes nanosheets for gas separation was achieved by Caro and coworkers where they exfoliated a MXene into 1.5-nm-thick nanosheets and assembled them, by simple vacuum filtration. ${ }^{[9]}$ The prepared 2- $\mu \mathrm{m}$-thick MXene-based membrane was self-standing due to the high in-plane elastic constant of MXenes, and the $0.35 \mathrm{~nm}$ simulated channels between the layers gave a $\mathrm{H}_{2}$ permeance $>2200$ barrer and a $\mathrm{H}_{2} / \mathrm{CO}_{2}$ selectivity over $160\left(1\right.$ barrer $\left.=3.3 \times 10^{-16} \mathrm{~mol} \mathrm{~m} \mathrm{~m}{ }^{-2} \mathrm{~s}^{-1} \mathrm{~Pa}^{-1}\right){ }^{[9]}$ Jin and coworkers developed a 20-nm-thick $\mathrm{Ti}_{3} \mathrm{C}_{2} \mathrm{~T}_{\mathrm{x}}$ MXene membrane and rationally designed the interlayer spacings with the intercalation of borate and $\mathrm{CO}_{2}$-philic agent changing the transport mechanism from a 'diffusion-controlled' to a 'solu- tion-controlled' type of transport.[65] They speculated that the transport was mainly through slit-like pores rather than nanochannels and their finding was supported by the fact that upon decrease of the gallery spacings from 0.52 to $0.38 \mathrm{~nm}$ the selectivity remained unchanged. Their functionalized membrane achieved a $\mathrm{CO}_{2}$ permeance of $350 \mathrm{GPU}$ with a $\mathrm{CO}_{2} / \mathrm{CH}_{4}$ selectivity of 15.3.[65]

The potential of synthesizing free-standing MXene membranes makes them very attractive since no expensive supports are needed. However, more investigation is needed to understand the molecule transport pathways and the relationship between interlayer spacing and the membrane performance.

\section{Conclusions}

The high performance achieved with 2D nanosheets-based membranes position them as state-of-art in many applications and motivates for further investigation. However, many challenges need to be addressed before 2D nanosheet-based membranes can be used in the industry. The current top-down exfoliation strategies cannot produce nanosheets of sufficient quality (i.e. large and uniform lateral size distribution), or the exfoliation yields are too low. On the other hand, the bottom-up approach is limited to small sizes and it is feasible only for limited cases.

Nanoporous graphene has triggered extensive interest due to its unique properties and could represent the next generation of membranes. However, pore size control, narrow pore size distribution, high pore density, and a more scalable transferring technique represent still substantial hurdles for its industrial application.

2D membranes as thin as few nanometers can be synthesized on a lab scale with very high performance, however, it remains challenging to engineer such films on a much larger industrial scale where small defects produced during processing may compromise the overall performance. Beyond the separation performance, investigations on how the membranes behave in real industrial operating conditions should be done (chemical and thermal stability, pressurized tests without sweep gas, mixed gas tests with real mixtures, etc.).

\section{Acknowledgements}

The author acknowledges the host institution, EPFL, for the generous support. This work was funded by Efficiency of Industrial Processes (SCCER-EIP). The author acknowledges his PI Professor K.V Agrawal and Dr. L.F. Villalobos for their insightful discussions about 2D materials for membranes.

\section{Conflict of interest}

The author declares no conflict of interest.

Received: March 7, 2020

[1] E. Drioli, A. I. Stankiewicz, F. Macedonio, J. Memb. Sci. 2011, 380, 1.

[2] A. Van Miltenburg, W. Zhu, F. Kapteijn, J. A. Moulijn, Chem. Eng. Res. Des. 2006, DOI 10.1205/cherd05021.

[3] D. S. Sholl, R. P. Lively, Nature 2016, 532, 435.

[4] H. B. Park, J. Kamcev, L. M. Robeson, M. Elimelech, B. D. Freeman, Science 2017, 356, eaab0530.

[5] K. S. Novoselov, A. K. Geim, S. V. Morozov, D. Jiang, Y. Zhang, S. V. Dubonos, I. V. Grigorieva, A. A. Firsov, Science 2004, 306, 666.

[6] G. Liu, W. Jin, N. Xu, Chem. Soc. Rev. 2015, 44, 5016.

[7] J. S. Bunch, S. S. Verbridge, J. S. Alden, A. M. Van Der Zande, J. M. Parpia, H. G. Craighead, P. L. McEuen, Nano Lett. 2008, 8, 2458.

[8] H. Li, Z. Song, X. Zhang, Y. Huang, S. Li, Y. Mao, H. J. Ploehn, Y. Bao, M. Yu, Science 2013, 342, 95.

[9] L. Ding, Y. Wei, L. Li, T. Zhang, H. Wang, J. Xue, L. X. Ding, S. Wang, J. Caro, Y. Gogotsi, Nat. Commun. 2018, 9, 1

[10] E. D. Grayfer, M. N. Kozlova, V. E. Fedorov, Adv. Colloid Interface Sci. 2017, 245,40 .

[11] Y. Liu, N. Wang, Z. Cao, J. Caro, J. Mater. Chem. A 2014, 2, 1235.

[12] S. Huang, M. Dakhchoune, W. Luo, E. Oveisi, G. He, M. Rezaei, J. Zhao, D. T. L. Alexander, A. Züttel, M. S. Strano, K. V. Agrawal, Nat. Commun. 2018, 9. 1 
[13] K. Varoon, X. Zhang, B. Elyassi, D. D. Brewer, M. Gettel, S. Kumar, J. A. Lee, S. Maheshwari, A. Mittal, C.-Y. Sung, M. Cococcioni, L. F. Francis, A V. McCormick, K. A. Mkhoyan, M. Tsapatsis, Science 2011, 334, 72.

[14] Y. Peng, Y. Li, Y. Ban, H. Jin, W. Jiao, X. Liu, W. Yang, Science 2014, 346, 1356.

[15] A. P. Côté, A. I. Benin, N. W. Ockwig, M. O'Keeffe, A. J. Matzger, O. M. Yaghi, Science 2005, 310, 1166.

[16] L. F. Villalobos, M. T. Vahdat, M. Dakhchoune, Z. Nadizadeh, M. Mensi, E. Oveisi, D. Campi, N. Marzari, K. V. Agrawal, Sci. Adv. 2020, 6, DOI 10.1126/sciadv.aay9851.

[17] Y. Peng, Y. Li, Y. Ban, H. Jin, W. Jiao, X. Liu, W. Yang, Science 2014, 346, 1356.

[18] G. Li, K. Zhang, T. Tsuru, ACS Appl. Mater. Interf. 2017, 9, 8433.

[19] X. Wang, C. Chi, K. Zhang, Y. Qian, K. M. Gupta, Z. Kang, J. Jiang, D. Zhao, Nat. Commun. 2017, 8, 14460.

[20] P. Z. Li, Y. Maeda, Q. Xu, Chem. Commun. 2011, 47, 8436.

[21] T. Rodenas, I. Luz, G. Prieto, B. Seoane, H. Miro, A. Corma, F. Kapteijn, F. X. Llabrés I Xamena, J. Gascon, Nat. Mater. 2015, 14, 48.

[22] R. Makiura, S. Motoyama, Y. Umemura, H. Yamanaka, O. Sakata, H. Kitagawa, Nat. Mater. 2010, 9, 565.

[23] Z. Chen, W. Chen, D. Jia, Y. Liu, A. Zhang, T. Wen, J. Liu, Y. Ai, W. Song, X. Wang, Adv. Sci. 2018, 5, 1800235 .

[24] A. M. Evans, L. R. Parent, N. C. Flanders, R. P. Bisbey, E. Vitaku, M. S. Kirschner, R. D. Schaller, L. X. Chen, N. C. Gianneschi, W. R. Dichtel, Science 2018, 361, 52.

[25] F. L. Li, P. Wang, X. Huang, D. J. Young, H. F. Wang, P. Braunstein, J. P. Lang, Angew. Chem. Int. Ed. 2019, 58, 7051.

[26] Q. Zheng, W. H. IP, X. Lin, N. Yousefi, K. K. Yeung, Z. Li, J.-K. Kim, ACS Nano 2011, 5, 6039.

[27] S. Xia, M. Ni, T. Zhu, Y. Zhao, N. Li, Desalination 2015, 371, 78.

[28] A. F. M. Ibrahim, Y. S. Lin, Chem. Eng. Sci. 2018, 190, 312.

[29] A. Akbari, P. Sheath, S. T. Martin, D. B. Shinde, M. Shaibani, P. C. Banerjee, R. Tkacz, D. Bhattacharyya, M. Majumder, Nat. Commun. 2016, 7, 1.

[30] N. Rangnekar, M. Shete, K. V. Agrawal, B. Topuz, P. Kumar, Q. Guo, I. Ismail, A. Alyoubi, S. Basahel, K. Narasimharao, C. W. Macosko, K. A. Mkhoyan, S. Al-Thabaiti, B. Stottrup, M. Tsapatsis, Angew. Chem. Int. Ed. 2015, 54, 6571.

[31] R. R. Nair, H. A. Wu, P. N. Jayaram, I. V. Grigorieva, A. K. Geim, Science 2012, 335, 442 .

[32] F. Zhou, H. N. Tien, W. L. Xu, J. T. Chen, Q. Liu, E. Hicks, M. Fathizadeh, S. Li, M. Yu, Nat. Commun. 2017, 8, 1.

[33] M. Tsapatsis, AIChE J. 2014, 60, 2374

[34] M. E. Davis, Nature 2002, 417, 813.

[35] J. Caro, M. Noack, P. Kölsch, Adsorption 2005, 11, 215.

[36] J. Choi, M. Tsapatsis, J. Am. Chem. Soc. 2010, 132, 448

[37] K. V. Agrawal, B. Topuz, T. C. T. Pham, T. H. Nguyen, N. Sauer, N. Rangnekar, H. Zhang, K. Narasimharao, S. N. Basahel, L. F. Francis, C. W. Macosko, S. Al-Thabaiti, M. Tsapatsis, K. B. Yoon, Adv. Mater. 2015, 27, 3243.

[38] D. Kim, M. Y. Jeon, B. L. Stottrup, M. Tsapatsis, Angew. Chem. Int. Ed. 2018, 57, 480 .

[39] M. Y. Jeon, D. Kim, P. Kumar, P. S. Lee, N. Rangnekar, P. Bai, M. Shete, B. Elyassi, H. S. Lee, K. Narasimharao, S. N. Basahel, S. Al-Thabaiti, W. Xu, H. J. Cho, E. O. Fetisov, R. Thyagarajan, R. F. De Jaco, W. Fan, K. A. Mkhoyan, J. I. Siepmann, M. Tsapatsis, Nature 2017, 543, 690.
[40] H. C. Zhou, J. R. Long, O. M. Yaghi, Chem. Rev. 2012, 112, 673.

[41] A. K. Cheetham, C. N. R. Rao, R. K. Feller, Chem. Commun. 2006, 4780.

[42] Y. Li, H. Liu, H. Wang, J. Qiu, X. Zhang, Chem. Sci. 2018, 9, 4132.

[43] S. Yuan, X. Li, J. Zhu, G. Zhang, P. Van Puyvelde, B. Van Der Bruggen, Chem. Soc. Rev. 2019, 48, 2665.

[44] Y. Wang, J. Li, Q. Yang, C. Zhong, ACS Appl. Mater. Interf. 2016, 8, 8694.

[45] M. Tong, Q. Yang, Q. Ma, D. Liu, C. Zhong, J. Mater. Chem. A 2015, 4, 124.

[46] H. Fan, A. Mundstock, A. Feldhoff, A. Knebel, J. Gu, H. Meng, J. Caro, J. Am. Chem. Soc. 2018, 140, 10094.

[47] Z. Kang, Y. Peng, Y. Qian, D. Yuan, M. A. Addicoat, T. Heine, Z. Hu, L. Tee, Z. Guo, D. Zhao, Chem. Mater. 2016, $28,1277$.

[48] V. Berry, Carbon N. Y. 2013, 62, 1.

[49] H. Liu, S. Dai, D. E. Jiang, Nanoscale 2013, 5, 9984.

[50] D. Cohen-Tanugi, J. C. Grossman, Nano Lett. 2012, 12, 3602.

[51] M. D. Fischbein, M. Drndic, Appl. Phys. Lett. 2008, 93, 113107

[52] K. Celebi, J. Buchheim, R. M. Wyss, A. Droudian, P. Gasser, I. Shorubalko, J. Il Kye, C. Lee, H. G. Park, Science 2014, 344, 289.

[53] S. P. Surwade, S. N. Smirnov, I. V. Vlassiouk, R. R. Unocic, G. M. Veith, S. Dai, S. M. Mahurin, Nat. Nanotechnol. 2015, 10, 459.

[54] S. P. Koenig, L. Wang, J. Pellegrino, J. S. Bunch, Nat. Nanotechnol. 2012, 7, 728.

[55] S. C. O’Hern, D. Jang, S. Bose, J. C. Idrobo, Y. Song, T. Laoui, J. Kong, R. Karnik, Nano Lett. 2015, 15, 3254.

[56] J. Zhao, G. He, S. Huang, L. F. Villalobos, M. Dakhchoune, H. Bassas, K. V. Agrawal, Sci. Adv. 2019, 5, DOI 10.1126/sciadv.aav1851.

[57] W. S. Hummers, R. E. Offeman, J. Am. Chem. Soc. 1958, 80, 1339.

[58] J. Shen, G. Liu, K. Huang, Z. Chu, W. Jin, N. Xu, ACS Nano 2016, 10, 3398.

[59] M. Deng, K. Kwac, M. Li, Y. Jung, H. G. Park, Nano Lett. 2017, 17, 2342.

[60] P. Cheng, Y. Chen, X. Yan, Y. Wang, W. Z. Lang, ChemSusChem 2019, 12, 275.

[61] D. Wang, Z. Wang, L. Wang, L. Hu, J. Jin, Nanoscale 2015, 7, 17649.

[62] A. Achari, S. Sahana, M. Eswaramoorthy, Energy Environ. Sci. 2016, 9, 1224.

[63] B. Anasori, M. R. Lukatskaya, Y. Gogotsi, Nat. Rev. Mater. 2017, $2,1$.

[64] M. Naguib, V. N. Mochalin, M. W. Barsoum, Y. Gogotsi, Adv. Mater. 2014, $26,992$.

[65] J. Shen, G. Liu, Y. Ji, Q. Liu, L. Cheng, K. Guan, M. Zhang, G. Liu, J. Xiong, J. Yang, W. Jin, Adv. Funct. Mater. 2018, 28, DOI 10.1002/adfm.201801511.

[66] M. Y. Jeon, 'Synthesis of Zeolite Nanosheets and Applications in Membranes and Adsorption Separation Processes', PhD dissertation, University of Minnesota, 2016.

\section{License and Terms}

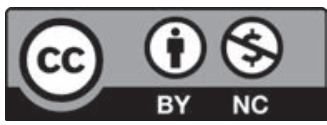

This is an Open Access article under the terms of the Creative Commons Attribution License CC BY_NC 4.0. The material may not be used for commercial purposes.

The license is subject to the CHIMIA terms and conditions: (http:// chimia.ch/component/sppagebuilder/?view=page \&id=12).

The definitive version of this article is the electronic one that can be found at doi:10.2533/chimia.2020.263 